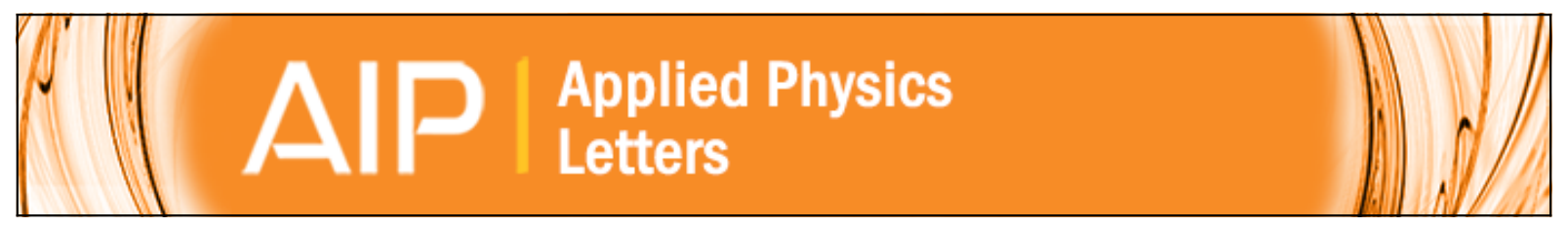

\title{
Electron counting of single-electron tunneling current
}

T. Fujisawa, T. Hayashi, Y. Hirayama, H. D. Cheong, and Y. H. Jeong

Citation: Applied Physics Letters 84, 2343 (2004); doi: 10.1063/1.1691491

View online: http://dx.doi.org/10.1063/1.1691491

View Table of Contents: http://scitation.aip.org/content/aip/journal/apl/84/13?ver=pdfcov

Published by the AIP Publishing

\section{Articles you may be interested in}

Transport properties of a quantum dot in quantum Hall regimes probed by a single-electron transistor Appl. Phys. Lett. 94, 232109 (2009); 10.1063/1.3155205

Fabrication of a germanium quantum-dot single-electron transistor with large Coulomb-blockade oscillations at room temperature

Appl. Phys. Lett. 85, 1532 (2004); 10.1063/1.1785870

Tunable negative differential resistance controlled by spin blockade in single-electron transistors

Appl. Phys. Lett. 80, 2177 (2002); 10.1063/1.1459489

Single-electron charging in a parallel dot structure

Appl. Phys. Lett. 78, 634 (2001); 10.1063/1.1344576

Fabrication of a single-electron transistor by current-controlled local oxidation of a two-dimensional electron system

Appl. Phys. Lett. 76, 457 (2000); 10.1063/1.125786

You don't

still use this

cell phone

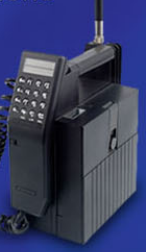

or this computer

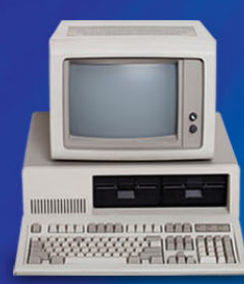

Why are you

still using an

AFM designed in the $80^{\prime}$ s?
It is time to upgrade your AFM Minimum \$20,000 trade-in discount for purchases before August 31st

Asylum Research is today's technology leader in AFM

dropmyoldAFM@oxinst.com

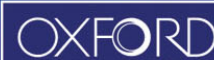




\title{
Electron counting of single-electron tunneling current
}

\author{
T. Fujisawa, ${ }^{\text {a) }}$ T. Hayashi, and Y. Hirayama ${ }^{\text {b) }}$ \\ NTT Basic Research Laboratories, NTT Corporation, 3-1 Morinosato-Wakamiya, Atsugi, 243-0198, Japan \\ H. D. Cheong \\ Hanbat National University, Duckmyoung-dong, Yusung-gu, Daejon, Korea \\ Y. H. Jeong \\ Pohang University of Science and Technology, Hyoja-dong, Nam-gu, Pohang, Kyungpook, Korea
}

(Received 3 November 2003; accepted 4 February 2004)

\begin{abstract}
Single-electron tunneling through a quantum dot is detected by means of a radio-frequency single-electron transistor. Poisson statistics of single-electron tunneling events are observed from frequency domain measurements, and individual tunneling events are detected in the time-domain measurements. Counting tunneling events gives an accurate current measurement in the saturated current regime, where electrons tunnel into the dot only from one electrode and tunnel out of the dot only to the other electrode. (C) 2004 American Institute of Physics. [DOI: 10.1063/1.1691491]
\end{abstract}

Single-electron tunneling is a correlated tunneling phenomenon that arises due to the Coulomb blockade (CB) effect, and is widely accepted in many physical systems. ${ }^{1}$ The correlation has been studied from ensemble characteristics, such as shot noise measurements and pumping current driven by an oscillating potential. ${ }^{2-4}$ However, direct observation of single-electron tunneling is remained in a very low frequency region. A radio-frequency single-electron transistor (RF-SET), in which charge on the island can be measured using an RF carrier signal reflected from or transmitted through the SET, ${ }^{5-7}$ allows us to detect each single-electron tunneling event through a quantum dot (QD) electrostatically coupled to the RF-SET. Because of its fast response and high sensitivity, this scheme should enable us to count tunneling electrons of an extremely small current accurately. Actually, this scheme has been demonstrated in a semiconductor QD coupled to a metal RF-SET. ${ }^{8}$ However, the measurement was performed in the thermally activated regime, where the count is not directly related to the current. In this letter, we report similar measurements on a semiconductor double QD in the saturated tunneling regime, where the count should correspond to the net current.

Figure 1(a) shows our experimental setup for counting single-electron tunneling events. Two electrically isolated SETs are integrated in an AlGaAs/GaAs heterostructure. Although the device is designed so that up to four QDs can be formed in the two channels, we activated one QD in each channel by applying negative gate voltages $V_{L}$ and $V_{R}$ to the upper SET and $V_{l}, V_{c}$, and $V_{r}$ to the lower one in this study. All measurements were performed at a temperature $T \sim 100$ $\mathrm{mK}$. The lower SET is combined with a $L C$ resonator (inductance $L=100 \mathrm{nH}$; capacitance $C \sim 0.6 \mathrm{pF}$ ) and works as a transmission-type RF-SET. ${ }^{6}$ An RF carrier signal at the resonant frequency (about $650 \mathrm{MHz}$ ) is introduced with an amplitude of about $0.3 \mathrm{mV}$ at the source electrode. The transmitted signal is amplified and detected as a voltage, $V_{\text {det }}$,

${ }^{a)}$ Author to whom correspondence should be addressed; electronic mail: fujisawa@will.brl.ntt.co.jp; also at Tokyo Institute of Technology.

${ }^{b)}$ Also at: SORST-JST, 4-1-8 Honmachi, Kawaguchi, 331-0012, Japan. which is proportional to the conductance of the lower SET. The resistance of the RF-SET is about $500 \mathrm{k} \Omega$ at the best charge sensitivity. The single-electron charging energy is about $1 \mathrm{meV}$ for the RF-SET, and about $1.5 \mathrm{meV}$ for the upper SET. The QD in each SET contains several ten electrons. The two QDs are separated by about $700 \mathrm{~nm}$ and electrostatically coupled with a small mutual capacitance. Adding an electron on the upper dot induces an equivalent charge of about $0.005 e$ on the lower dot and changes the RF-SET
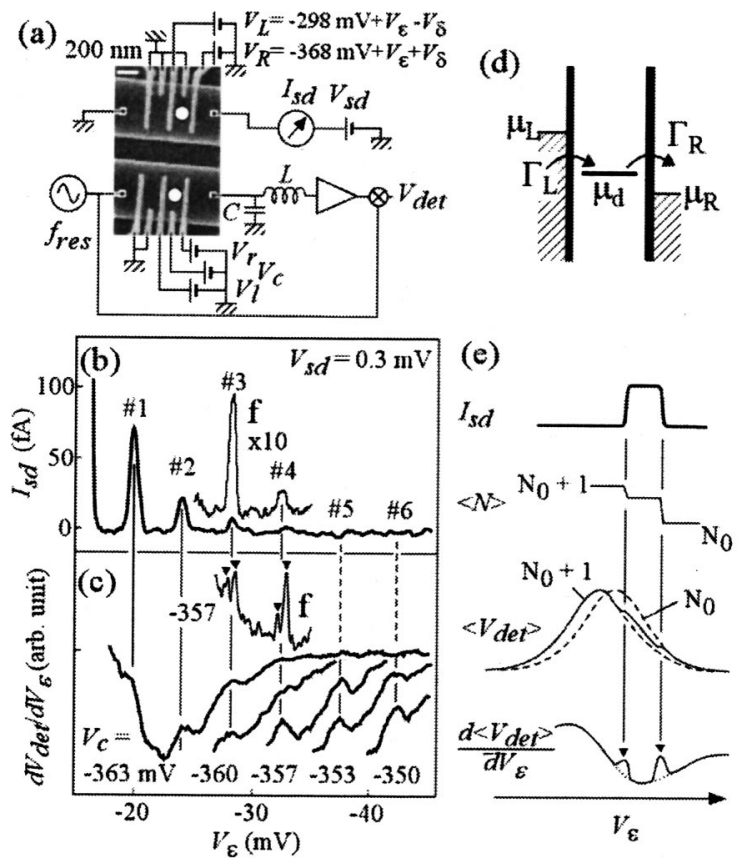

FIG. 1. (a) Schematic diagram of the measurement setup. The sample shown in the scanning electron micrograph contains two GaAs QDs (white circles) made by dry etching (upper, central, and lower dark regions) and Schottky gates (vertical bright lines). The measurement was performed in a dilution refrigerator $(T \sim 0.1 \mathrm{~K})$. (b) Typical Coulomb blockade oscillations of the upper QD measured by a current. (c) Derivative of the RF-SET signal, $d\left\langle V_{\text {det }}\right\rangle / d V_{\epsilon}$. The fine trace, labeled $\mathrm{f}$, is offset vertically for clarity. (d) Energy diagram of a single-electron tunneling process. (e) Schematic illustration of $I_{\text {sd }},\langle N\rangle,\left\langle d V_{\text {det }}\right\rangle$ and $d\left\langle V_{\text {det }}\right\rangle / d V_{\epsilon}$ around a CB peak in the saturated current regime. 
signal $V_{\text {det }}$. This coupling is much weaker than that used in previous studies ${ }^{6,8}$ and therefore effectively minimizes the heating effect on the upper QD from the RF signal.

We start from static characteristics of the upper SET. In our device, tunneling rates, $\Gamma_{L}$ and $\Gamma_{R}$ respectively for the left and right barriers, can be controlled by $V_{L}$ and $V_{R}$. For convenience, we define $V_{\epsilon}\left(V_{\delta}\right)$, which changes $V_{L}$ and $V_{R}$ simultaneously in the same direction (in the opposite direction) [defined in Fig. 1(a)]. Increasing $V_{\epsilon}$ lowers the potential of the upper QD and increases $\Gamma_{L}$ and $\Gamma_{R}$ with the ratio $\Gamma_{L} / \Gamma_{R}$ kept almost constant. Increasing $V_{\delta}$ increases the ratio $\Gamma_{L} / \Gamma_{R}$ with the dot potential kept almost constant. Figure 1(b) shows Coulomb blockade oscillations that appeared in the current $I_{\mathrm{sd}}$ through the upper SET when $V_{\epsilon}$ was swept at the symmetric barrier condition, $\Gamma_{L} \sim \Gamma_{R}$, at $V_{\delta}=0$. The peak current decreases dramatically as $\Gamma_{L}$ and $\Gamma_{R}$ decrease. During this measurement, the averaged RF-SET signal $\left\langle V_{\text {det }}\right\rangle$ was simultaneously monitored. Its derivative $d\left\langle V_{\text {det }}\right\rangle / d V_{\epsilon}$ is plotted in Fig. 1(c). Since the gate voltages for the upper SET slightly affect the RF-SET, we adjusted $V_{c}$ to obtain sufficient charge sensitivity for the whole $V_{\epsilon}$ range. One can see small peaks (Nos. 1-6) in the $d\left\langle V_{\operatorname{det}}\right\rangle / d V_{\epsilon}$ curves at the same $V_{\epsilon}$ where current peaks appear in $I_{s d}$, as indicated by vertical lines. Single-electron charging on the upper QD is reflected in the conductance through the lower QD. ${ }^{8,9}$

In a fine sweep, labeled "f" in Fig. 1(c), however, each peak divided into two peaks (indicated by triangles). This is the signature of the saturated tunneling regime. When a relatively large source-drain voltage, $V_{\mathrm{sd}}$, is applied as shown in the energy diagram of Fig. 1(d), an electron enters the dot with a rate $\Gamma_{L}$ and leaves the dot with a rate $\Gamma_{R}$. For simplicity, the electrochemical potential $\mu_{d}$ for only the ground state is considered. When $\mu_{d}$ is located between the electrochemical potential of the left and right electrodes, $\mu_{L}$ and $\mu_{R}$ respectively, and the separation between $\mu_{d}, \mu_{L}$, and $\mu_{R}$ is much larger than thermal energy, $k T$, and linewidth, $\hbar \Gamma$, the current is given by $I=\mathrm{e} \Gamma_{L} \Gamma_{R} /\left(\Gamma_{L}+\Gamma_{R}\right)$ independent of other parameters, such as $V_{\mathrm{sd}} .{ }^{10}$ In this case, backward tunneling processes, i.e., tunneling from the QD to the left electrode, are blocked. This is the situation where the count of single-electron tunneling events should agree with the net current amplitude. Outside this regime, the count increases by the number of backward tunneling events, since charge measurement cannot determine which electrode an electron has tunneled to. ${ }^{8}$

In this saturated current regime, a CB current peak has a broad flat-top current profile, as shown by the uppermost curve in Fig. 1(e). The average electron number in the constant current region is given by $\langle N\rangle=N_{0}+\Gamma_{L} /\left(\Gamma_{L}+\Gamma_{R}\right)$, as illustrated by the second curve. Due to the coupling between the two QDs, the average value of $V_{\text {det }}$ exhibits two steps superimposed in a broad CB peak (the third curve), and two corresponding peaks arises in the derivative $d\left\langle V_{\text {det }}\right\rangle / d V_{\epsilon}$ (the lowest curve). Thus, the saturated current region is located between the two peaks.

Figure 2(a) shows the $d\left\langle V_{\text {det }}\right\rangle / d V_{\epsilon}$ traces observed when the symmetry of the tunneling rates were changed. Since a larger $V_{\text {sd }}$ of $0.5 \mathrm{mV}$ was applied, the double peak characteristics were clearly resolved, but only observed at $V_{\delta}=0$, where the two barriers are almost symmetric $\left(\Gamma_{L} \sim \tilde{\Gamma}_{R}\right)$.

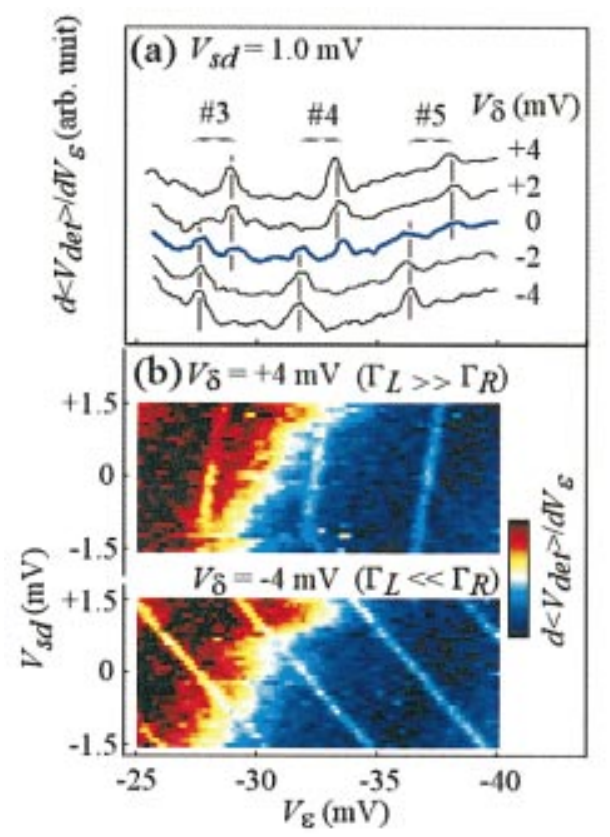

FIG. 2. (Color) (a) $d\left\langle V_{\text {det }}\right\rangle / d V_{\epsilon}$ traces when the symmetry of the barriers is changed by $V_{\delta}$. (b) Color intensity plots of $d\left\langle V_{\mathrm{det}}\right\rangle / d V_{\epsilon}$ for two asymmetricbarrier conditions.

When the barriers are strongly asymmetric, $\langle N\rangle$ becomes close to an integer, and only one peak can be observed. Figure 2(b) shows the intensity plots of $d\left\langle V_{\text {det }}\right\rangle / d V_{\epsilon}$ for two asymmetric-barrier conditions. The peaks move on the condition $\mu_{L}=\mu_{d}$ for $\Gamma_{L} \gg \Gamma_{R}$ in the upper plot and on $\mu_{d}$ $=\mu_{R}$ for $\Gamma_{L} \ll \Gamma_{R}$ in the lower plot. This also supports the above discussions about the saturated transport regime.
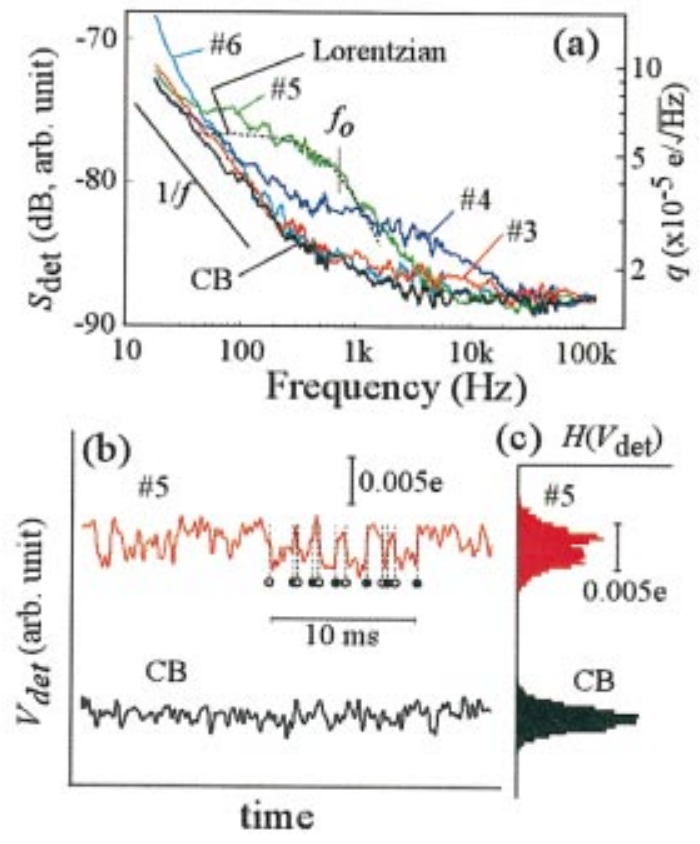

FIG. 3. (Color) (a) Frequency spectra of the RF-SET signal, $V_{\text {det }}$, for the $\mathrm{CB}$ condition and single-electron transport conditions (Nos. 3-6). Monochromatic peaks $(50-\mathrm{Hz}$ harmonics) have been removed for better visibility. The dashed line is a Lorentzian fitting to spectrum No. 5. (b) Time-domain measurements of $V_{\text {det }}$ for CB (the lower trace) and the single-electron tunneling regime (the upper trace for No. 5). No averaging was performed (c) Histograms, $H\left(V_{\text {det }}\right)$, of the two traces of (b) but for a longer time domain. 
When excited states are involved in the transport, $\langle N\rangle$ is determined by the total tunneling rate to ground and excited states, and usually excited states have higher tunneling rates. Therefore, the effective total tunneling rates may become asymmetric. In order to minimize the influence from excited states, we performed the following measurements at $V_{\text {sd }}=0.3$ $\mathrm{mV}$, which is comparable to or even smaller than typical energy spacing of $0.3-0.5 \mathrm{meV}$ for the upper QD. We confirmed well-isolated double peak structures in fine sweeps. Note again that the double peak is convincing evidence of the saturated current regime, even though the current is too small to see.

Now we discuss the time-dependent part of the charge detection. Figure 3(a) shows the power spectra, $S_{\text {det }}(f)$, of $V_{\text {det }}$. The corresponding equivalent charge on the lower QD, $q$, is shown in the right scale. The black trace, labeled CB, was obtained when the upper SET was in the Coulomb blockade region, and indicates the background noise in our measurement system including the RF-SET. The lowfrequency $1 / f$ noise originates from the QD device, while the high-frequency white noise comes from the cold RF amplifier. ${ }^{6}$ Other traces, labeled Nos. 3-6, were measured at $V_{\delta}=0$ in the saturated current regime. The excess spectrum above the background noise can be expressed by $S_{q}(f)$ $=A^{2}\left(e^{2} / 2 \pi^{2}\right) \Gamma /\left[f^{2}+(\Gamma / \pi)^{2}\right]$, which is expected for singleelectron tunneling current with symmetric tunneling rates, $\Gamma_{L}=\Gamma_{R}=\Gamma{ }^{11} A \sim 0.005$ is the coupling coefficient between the two dots. The characteristic (3-dB rolloff) frequency, $f_{0}=\Gamma / \pi$, changes consistently with the tunneling current shown in Fig. 1(b). The fitting to the curve for No. 5 was performed using parameters $f_{0}=750 \mathrm{~Hz}$ and $A=0.006$ (the dashed line). The spectrum for peak No. 4 shows $\Gamma \sim 30 \mathrm{kHz}$ $\left(f_{0} \sim 10 \mathrm{kHz}\right)$, which is almost the same as the $\Gamma \sim 20 \mathrm{kHz}$ estimated from the peak current of $1.5 \mathrm{fA}$ in Fig. 1(b). This coincidence clearly indicates that the observed excess spectrum comes from single-electron tunneling current in the saturated current regime.

Figure 3(b) shows typical $V_{\text {det }}$ traces in the time domain. We used a $1-\mathrm{kHz}$ first-order low-pass filter to cut highfrequency noise. The lower trace was measured when the upper QD was in the $\mathrm{CB}$ regime, and indicates the background noise. The upper trace measured for peak No. 5 includes single-electron tunneling events, at which $V_{\text {det }}$ goes up (down) when an electron leaves (enters) the dot as marked by solid (open) circles. The amplitude of the switching signal corresponds to the equivalent charge of $0.005 e$ on the lower $\mathrm{QD}$, which is consistent with the static characteristics. Unfortunately, the tunneling events are not always clear because of the insufficient signal-to-noise ratio. Typical switching frequency for the marks is about $\Gamma=1 \mathrm{kHz}$, which corresponds to about $0.1 \mathrm{fA}$. This $\Gamma$ is somewhat smaller than the $\Gamma \sim 2.4 \mathrm{kHz}\left(f_{0} \sim 750 \mathrm{~Hz}\right)$ obtained from the frequency spectrum, probably because some short-period tunneling events are filtered out. Although the accuracy of the counting is not very good at present, it is clear that each tunneling event corresponds to an electron flow through a QD. It should be noted that measuring sub-femtoampere current in a $10 \mathrm{~ms}$ period is extremely sensitive as compared to measurement with a conventional current meter.

The single-electron tunneling correlation can be seen in the histogram for $V_{\text {det }}$ as shown in Fig. 3(c). The appearance of two peaks for No. 5 proves the single-electron tunneling scheme, in which only two charge states are allowed. However, a single peak can be seen in the histogram in the CB regime, where the number of electrons is fixed at an integer.

We have successfully observed single-electron tunneling events in both frequency and time domains. The accuracy of electron counting can be improved by increasing the coupling coefficient and decreasing the background noise in the measurement system. For the present result, the count should correspond to the saturated current, which is given by device parameters $\left(\Gamma_{L}\right.$ and $\left.\Gamma_{R}\right)$. In order to count electrons for arbitrary current, the upper QD can be replaced with a weakly coupled double quantum dot. Since electrons would then be transferred through three charge states, the direction of the electron flow could also be determined. Our device is also designed for this purpose. Furthermore, this technique can be applied to detect charge states and spin states of a single or double quantum dot in a short time. ${ }^{12,13}$ If the measurement time for detecting an electron can be made shorter than the energy relaxation time of the system, the quantum state can be measured at a time (single shot measurement). The highspeed charge detection scheme would bring about various classical and quantum information technologies.

This work was partially supported by the Ministry of Education of Korea through its BK21 program.

${ }^{1}$ Single Charge Tunneling, Coulomb Blockade Phenomena in Nanostructures, NATO ASI, Ser. B., edited by H. Grabert and M. H. Devoret (Plenum, New York, 1992), Vol. 294.

${ }^{2}$ L. I. Glazman and K. A. Matveev, JETP Lett. 48, 445 (1988).

${ }^{3}$ H. Birk, M. J. M. de Jong, and C. Shonenberger, Phys. Rev. Lett. 75, 1610 (1995).

${ }^{4}$ P. Delsing, K. K. Likharev, L. S. Kuzmin, and T. Claeson, Phys. Rev. Lett. 63, 1861 (1989).

${ }^{5}$ R. J. Schoelkopf, P. Wahlgren, A. A. Kozhevnikov, P. Delsing, and D. E. Prober, Science 280, 1238 (1998).

${ }^{6}$ T. Fujisawa and Y. Hirayama, Appl. Phys. Lett. 77, 543 (2000).

${ }^{7}$ H. D. Cheong, T. Fujisawa, T. Hayashi, Y. Hirayama, and Y. H. Jeong, Appl. Phys. Lett. 81, 3257 (2002).

${ }^{8}$ W. Lu, Z. Ji, L. Pfeiffer, K. W. West, and A. J. Rimberg, Nature 423, 422 (2003).

${ }^{9}$ S. Gardelis, C. G. Smith, J. Cooper, D. A. Ritchie, E. H. Linfield, Y. Jin, and M. Pepper, Phys. Rev. B 67, 073302 (2003).

${ }^{10}$ H. van Houten, C. W. J. Beenakker, and A. A. M. Staring, in Single Charge Tunneling, Coulomb Blockade Phenomena in Nanostructures, NATO ASI, Ser. B., edited by H. Grabert and M. H. Devoret (Plenum, New York, 1991), Vol, 294, pp. 167-216.

${ }^{11}$ M. J. Kirton and M. J. Uren, Adv. Phys. 38, 367 (1989).

${ }^{12}$ A. Aassime, G. Johansson, G. Wendin, R. J. Schoelkopf, and P. Delsing, Phys. Rev. Lett. 86, 3376 (2001).

${ }^{13}$ J. M. Elzerman, R. Hanson, J. S. Greidanus, L. H. Willems van Beveren, S. De Franceschi, L. M. K. Vandersypen, S. Tarucha, and L. P. Kouwenhoven, Phys. Rev. B 67, 161308 (2003). 